\title{
DISCUSSION: Final Stages, Nucleosynthesis
}

\author{
Norbert Langer, Chairman \\ Astronomical Institute, P.O. Box 80000, NL-3508 TA Utrecht The \\ Netherlands
}

Many contributions to this conference have shown that its angular momentum can be as important to the evolution of a star as its mass or chemical composition. The preceding session has revealed large progress in our understanding of the angular momentum evolution of stars of various masses, which - extremely simplified - can be summarised in the following table.

\begin{tabular}{c|c|cc|c}
\multicolumn{5}{c}{ Specific angular momenta $/ \mathrm{cm}^{2} \mathrm{~s}^{-1}$} \\
\hline & ZAMS & $\begin{array}{c}\text { end } \\
\text { obs. }\end{array}$ & $\begin{array}{c}\text { stage } \\
\text { mod. }\end{array}$ & rigid rot. \\
\hline $2 \ldots 8 \mathrm{M}_{\odot}$ & $10^{18}$ & WD: $10^{14}$ & $10^{15}$ & $10^{10}$ \\
$>8 \mathrm{M}_{\odot}$ & $10^{18}$ & NS: $10^{14}$ & $510^{14}$ & $10^{10}$ \\
\hline \hline
\end{tabular}

It appears as if most stars above $2 \mathrm{M}_{\odot}$ (those of lower mass have been extensively discussed in previous sessions) start out with $j \simeq 10^{18} \mathrm{~cm}^{2} \mathrm{~s}^{-1}$ and end at $j \simeq 10^{14} \mathrm{~cm}^{2} \mathrm{~s}^{-1}$ - independent of whether they become white dwarfs or neutron stars. A specific angular momentum of $10^{14} \mathrm{~cm}^{2} \mathrm{~s}^{-1}$ is (logarithmicly) about half way between local angular momentum conservation and completely rigid rotation. The recently obtained rotating evolutionary models produce typically stellar remnants which rotate one to two orders of magnitude faster, unless, for the massive stars, magnetic fields are included in the interior models. This gives rise to two questions:

1. Do we need to include magnetic field to understand the internal angular momentum evolution of stars?

2. Can some massive stars, despite the evidence from recent models and from observations of compact stars, end with $10^{16} \mathrm{~cm}^{2} \mathrm{~s}^{-1}$, in order to produce gamma-ray bursts from collapsars and highly aspherical supernovae?

Woosley: White dwarf progenitors are likely to slow down due to their internal magnetic fields and shear effects. But question 2 means: can we produce a Wolf-Rayet star which is rotating rapidly at the time of core collapse?

Maeder: The requirement of rapidly rotating Wolf-Rayet stars seems to leave little room for magnetic effects in their progenitors. However, Wolf-Rayet stars lose mass at a high rate, and it turns out to be very difficult to avoid considerable angular momentum loss at the same time. 
Langer: As we need just a minority of stars to end their lives with a rapidly spinning core, we might leave the problem to achieve this to binaries: The accreting star in a mass transferring binary is dramatically spun-up.

Owocki: We might wonder what kind of Wolf-Rayet mass loss and magnetic field effects are needed.

Maeder: To answer this we need to follow new evolutionary calculations which account for the anisotropic mass loss due to stellar winds.

Christensen-Dalsgaard: It would be interesting to know whether the effect of magnetic fields inside stars is metallicity dependent.

Herwig: It appears to me that intermediate mass stars do not require a large effect of magnetic fields to slow down their cores, according to the current nonmagnetic rotating evolutionary models.

Heger: I agree that it is difficult to have a strong mass loss and little angular momentum loss in single stars. Might their be ways to avoid this coupling in massive close binary systems?

Langer: I think that having mass loss without angular momentum loss is also very difficult for binary components, as having mass gain without angular momentum gain (see below).

Collins: One potentially important possibility to obtain rapidly rotating stars has not been mentioned yet: stellar mergers.

Woosley: To explain the gamma-ray bursts, binary stars might indeed be needed, and stellar mergers are being discussed as one possibility. The problem is that we need to spin-up the core, and it is currently unknown whether angular momentum, of which we have plenty in a merger envelope, can reach the core or not.

Spruit: It is indeed so that mean molecular weight gradients reduce or prevent angular momentum transport, for the rotationally induced hydrodynamic instabilities as for magnetic mechanisms.

A further question of fundamental physical importance, which we have encountered ever again during the preceding session, can be phrased as follows:

3. How does the angular momentum of a star change when it loses or gains mass, i.e., what is the possible range of $\dot{J} / \dot{M}$ ? More specifically: Is it inevitable that a non-magnetic, rapidly rotating star which loses mass to a stellar wind spins down, or can it even spin-up under certain conditions? And: Is it inevitable that a non-magnetic accreting star spins up?

Heger: In principle, an anisotropic wind can spin the star up, if the latitudeaverage specific angular momentum in the wind is smaller than the average surface specific angular momentum; however it requires an extreme concentration of the mass loss towards the pole.

Owocki: In the last years we have seen that hot rotating stars may in fact, counter-intuitively, have a wind outflow concentrated toward the stellar rotation axis, which is largely a consequence of the von Zeipel effect: most photons leave the star through the hot polar surface regions rather than through the cooler equatorial regions.

Spruit: Mass gain without angular momentum gain for non-magnetic stars has been shown to be possible: just as in accretion disks, angular momentum can be transported outwards through viscosity in stars, and thus reach the accretion 
disk. Non-rotating stars would still be spun-up by disk-accretion, but angular momentum does not necessarily limit the accretion. However, realistic stars often do have magnetic fields, and it is worth pointing out in this context that we know of accreting neutron stars systems where the neutron star is being spun-down. 